\title{
Effect of annealing temperature on optical and electrical properties of metallophthalocyanine thin films deposited on silicon substrate
}

\author{
R. SKONIECZnY ${ }^{1}$, P. Popielarski ${ }^{3}$, W. BAŁA ${ }^{3}$, K. FABISIAK $^{3}$, K.PAProcki $^{3}$, \\ M. JANCELEWICZ ${ }^{2}$, M. KOWALSKA ${ }^{4}$, M. SZYBOWICZ ${ }^{1, *}$ \\ ${ }^{1}$ Faculty of Technical Physics, Poznan University of Technology, Piotrowo 3, 60-965 Poznan, Poland \\ ${ }^{2}$ Nanobiomedical Center, Adam Mickiewicz University, Umultowska 85, 61-614 Poznan, Poland \\ ${ }^{3}$ Institute of Physics, Kazimierz Wielki University, Powstańców Wielkopolskich 2, 85-090 Bydgoszcz, Poland \\ ${ }^{4}$ Faculty of Chemical Technology and Engineering, University of Technology and Life Sciences, \\ Seminaryjna 3, 85-326, Bydgoszcz, Poland
}

\begin{abstract}
The cobalt phthalocyanine (CoPc) thin films (300 nm thick) deposited on n-type silicon substrate have been studied using micro-Raman spectroscopy, atomic force spectroscopy (AFM) and I-V measurement. The CoPc thin layers have been deposited at room temperature by the quasi-molecular beam evaporation technique. The micro-Raman spectra of CoPc thin films have been recorded in the spectral range of $1000 \mathrm{~cm}^{-1}$ to $1900 \mathrm{~cm}^{-1}$ using $488 \mathrm{~nm}$ excitation wavelength. Moreover, using surface Raman mapping it was possible to obtain information about polymorphic forms distribution (before and after annealing) of metallophthalocyanine ( $\alpha$ and $\beta$ form) from polarized Raman spectra. The I-V characteristics of the Au/CoPc/n-Si/Al Schottky barrier were also investigated. The obtained results showed that influence of the annealing process plays a crucial role in the ordering and electrical conductivity of the molecular structure of CoPc thin films deposited on n-type silicon substrate.
\end{abstract}

Keywords: copper phthalocyanine; Raman spectroscopy; molecular orientation; atomic force microscopy; heat treatment

(C) Wroclaw University of Technology.

\section{Introduction}

Metallophthalocyanines are p-type semiconductors which possess very interesting optical and electrical properties. Due to their low cost of production, high thermal and chemical stability, metallophthalocyanines, especially in the form of thin films, can be applicable in many advanced technological devices [1-3]. Thin layers of metallophthalocyanines (MPc) as well as their junctions with a number of organic and inorganic semiconductors or metals can be applicable in optoelectronics as for example light-emitting diodes, photovoltaic cells, and organic field effect transistors. Charge injection at the interface between metallic electrodes and organic semiconductors are crucial for

*E-mail: miroslaw.szybowicz@put.poznan.pl the performance of organic optoelectronic devices. Moreover, the metallophthalocyanines are very useful as transporting and injection layers in optoelectronic devices $[4,5]$. For this reason the knowledge of their optical and electrical properties is very important.

Due to these exceptional properties, thin films of metallophthalocyanine have been recently intensively investigated especially by the optical methods such as absorption, ellipsometry, harmonic generation and Raman spectroscopy [6-11]. The Raman spectroscopy can be a useful tool to study various intra- and intermolecular energy transfer processes.

In order to improve devices technology, the deposition technique must provide a film with reproducible electrical and optical response characteristics. 
In case of CoPc thin films, the knowledge of the surface morphology and the preferred orientation of the crystallites and their electrical parameters are essential for potential successful applications. The preferred orientation is usually determined by sample preparation conditions such as type of substrate, method of evaporation and deposition temperature. Very important are also heating and cooling processes of organic thin layers, which in many cases lead to the changes in polymorphic forms and structure arrangement, changing the physical properties of the layer. In case of $\mathrm{CoPc}$ the structure at room temperature is determined by flat molecules with $\mathrm{D}_{4 \mathrm{~h}}$ symmetry [12]. The heating process changes the polymorphic form of the layer changing also its physical, electrical and optical properties.

In this paper, we present the results of the research on the structural orientation and electrical parameters of CoPc thin films deposited on silicon substrate.

The main aim of our work was to determine the CoPc molecular orientation and distribution of polymorphic phases. The electrical parameters of $\mathrm{p}-\mathrm{n}$ junction ( $\mathrm{p}-\mathrm{CoPc} / \mathrm{n}-\mathrm{Si}$ - organic diode) were also investigated.

\section{Experimental}

\subsection{Preparation of the thin films}

The cobalt phthalocyanine $(\mathrm{CoPc})$ layers have been prepared in a vacuum chamber at a pressure of about $\mathrm{p}=2 \times 10^{-4} \mathrm{~Pa}$. Purified CoPc powder (97\% Sigma Aldrich, Co.) was loaded onto a quartz effusion cell with a nozzle of $3 \mathrm{~mm}$ in di-

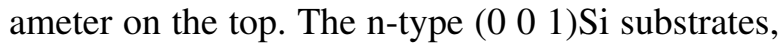
which before loading into the vacuum chamber, were sonificated in acetone, distilled water, isopropanol, and finally in distilled water. During evaporation process the $\mathrm{Si}$ substrates were held at room temperature. The deposition rate of thin $\mathrm{CoPc}$ layers was $0.2 \mathrm{~nm} / \mathrm{s}$.

\subsection{Raman scattering spectroscopy}

The Raman scattering spectra (RS) of cobalt phthalocyanine $(\mathrm{CoPc})$ were recorded by Renishaw inVia micro-Raman system. As an excitation source, a $488 \mathrm{~nm}$ wavelength from ion argon laser was used. The spectral range was chosen from approximately $1000 \mathrm{~cm}^{-1}$ to $1800 \mathrm{~cm}^{-1}$. Two types of polarized RS were collected in the scattering geometry VV and VH. Motorized stages were used to perform Raman surface mapping measurements. The laser beam was focused on the sample by a Leica microscope objective (magnification $\times 100$ ) with numerical aperture (NA) equal to 0.85 . Those parameters correspond to laser spot diameter about $1 \mu \mathrm{m}$. To prevent any damages caused by excessive excitation power, the power was fixed at $1 \mathrm{~mW}$. Raman maps were measured on a surface of $20 \mu^{2} \times 20 \mu \mathrm{m}^{2}$ with a step of $2 \mu \mathrm{m}$. Because all measurements were recorded in backscattering geometry we could neglect the angle dependence of reflection coefficients of both thin layer and substrate for different polarizations of the incident light.

\subsection{Atomic force spectroscopy}

Atomic Force Microscopy (AFM) images were performed with use of Catalyst Bruker Nanoscope. Height images were recorded using silicon nitride cantilevers with a spring constant of $0.4 \mathrm{~N} / \mathrm{m}$ and resonant frequency $70 \mathrm{kHz}$. All images were recorded in tapping mode and at room temperature.

\subsection{I-V measurements}

Current-voltage measurements (I-V characteristics) were carried out using Keitley 6485 picoammeter within voltage range $-5 \mathrm{~V}$ to $+5 \mathrm{~V}$ using homemade software to support the picoammeter voltage source and collection of measured I-V data. I-V characteristics were carried out at different temperatures in the range of $210 \mathrm{~K}$ to $440 \mathrm{~K}$. During electrical measurements organic thin layer was electrically insulated from external conditions.

\section{Results and discussion}

It has been shown that the orientation of metal phthalocyanine thin films relatively to the surface can be determined by different approaches. The angle of inclination of a molecule plane to the substrate can be resolved by electron diffraction 
techniques or high resolution transmission electron microscopy (HRTEM). Polarization Raman study of films allows one to define the structural parameters (polymorphism of the molecules). As the advantages of Raman spectroscopy, high spatial resolution, absence of substrate influence and lack of requirements on sample preparation can be mentioned.

At room temperature $\mathrm{CoPc}$ molecule is planar and possesses $\mathrm{D}_{4 \mathrm{~h}}$ point group symmetry. The determination of orientation of phthalocyanine molecules layer from Raman scattering measurements was based on the analysis of external molecular vibration. The method which allows one to determine the inclination angle of the molecules of phthalocyanine to the substrate was proposed by Kolesov [9].

The vibration of $\mathrm{CuPc}$ molecule consisting of 57 atoms and possessing $\mathrm{D}_{4 \mathrm{~h}}$ point group symmetry can be classified into the following irreducible representation $[9,14]$ :

$$
\begin{aligned}
\Gamma_{v i b}= & 14 A_{1 g}+13 A_{2 g}+14 B_{1 g}+14 B_{2 g}+13 E_{g} \\
& +6 A_{1 u}+8 A_{2 u}+7 B_{1 u}+7 B_{2 u}+28 E_{u}
\end{aligned}
$$

where $A_{1 g}, B_{1 g}, B_{2 g}$ and $E_{g}$ modes are Ramanactive. The nondegenerate $A_{1 g}, B_{1 g}, B_{2 g}$ modes are in-plane vibrations, and double degenerate $\mathrm{E}_{\mathrm{g}}$ are out-of-plane vibrations.

It is crucial to know the $\mathrm{IVV}_{\mathrm{VV}} / \mathrm{I}_{\mathrm{VH}}$ ratio of symmetry types vibrations $\left(\mathrm{A}_{1 \mathrm{~g}}, \mathrm{~B}_{1 \mathrm{~g}}\right)$ to estimate the molecular orientation (polymorphic form of molecules/CoPc), where $\mathrm{I}_{\mathrm{VV}}$ and $\mathrm{I}_{\mathrm{VH}}$ are integral intensities of Raman scattering for vertical and horizontal polarization of scattered light, respectively. The inclination angle of the molecule plane to the substrate plane is calculated from intensities ratio $\mathrm{I}_{\mathrm{VV}} / \mathrm{I}_{\mathrm{VH}}$ for $\mathrm{B}_{1 \mathrm{~g}}$ Raman mode achieved from Raman spectra. The detailed description has been given in our previous works [13-15]. The angle $\beta$ is a parameter representing the angle between molecule plane and substrate surface. This can be obtained from the following formula:

$$
\frac{I_{V V}}{I_{V H}}=2 \frac{\cos ^{2} \beta}{\sin ^{2} \beta}
$$

The molecular orientation of $\mathrm{CoPc}$ thin film was investigated for a film with the thickness equal to $300 \mathrm{~nm}$. Fig. 1 shows the polarized Raman spectra (VV and $\mathrm{VH}$ ) of CoPc $300 \mathrm{~nm}$ thin film on silicon substrate obtained before annealing process.

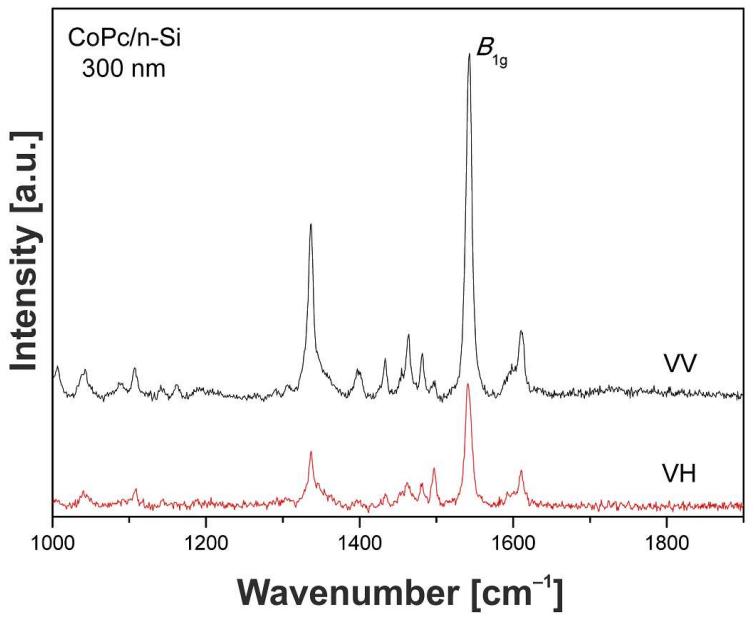

Fig. 1. Polarized Raman spectra (VV and VH) for CoPc thin layer deposited on Si substrate.

The position of the most intense band $\left(\mathrm{B}_{1 \mathrm{~g}} \mathrm{Ra}-\right.$ man mode) is $1542 \mathrm{~cm}^{-1}$ and is associated with the cobalt ion $\left(\mathrm{Co}^{2+}\right)$ adapted to the phthalocyanine molecule and can be designated by the displacement of $\mathrm{C}-\mathrm{N}-\mathrm{C}$ bridge bonds of molecule macrocycle. From the previous study it is known that for different metal atoms substituted in the center of phthalocyanine the energy of $\mathrm{B}_{1 \mathrm{~g}}$ band is related to the mass of the metal atom, metal ion size and diameter of phthalocyanine cavity [13-15]. In Fig. 1, it can be seen that the intensity of Raman spectrum recorded at parallel polarization of scattered light (VV) is approximately 3 times higher than for the spectrum recorded at cross polarization $(\mathrm{VH})$.

In order to determine and assess the distribution of inclination angle of molecules surface, Raman mapping measurements were performed before and after the annealing process. The procedure was conducted at temperature $473 \mathrm{~K}$ for 6 hours in air condition. Each map (Fig. 2) covers an area of $20 \mu \mathrm{m}^{2} \times 20 \mu \mathrm{m}^{2}$, and consists of 121 point measurements (done every $2 \mu \mathrm{m}$ ). Using numerical procedure, each spectrum was fitted to the theoretical Lorentz curve (using Wire 3.0 inVia software) 
to obtain integral intensity of $\mathrm{B}_{1 \mathrm{~g}}$ pyrrole stretch Raman mode at $1542 \mathrm{~cm}^{-1}$. Using relation 2 the intensities ratio and then the angle between molecule and substrate were calculated. An average angle of inclination for CoPc $300 \mathrm{~nm}$ film before annealing process was calculated from the map (Fig. 2a) and was $41.35^{\circ}$. After the annealing procedure (Fig. 2b) the angle of inclination slightly decreased to the value of $41.15^{\circ}$. Both values are very close to each other and when the standard deviation is taken into account, it can be assumed that they are equal. The estimated values of the inclination angles show that CoPc $300 \mathrm{~nm}$ film deposited on silicon had the $\beta$ polymorphic form before and after annealing ( $\beta$-form is equal to $40^{\circ}$ to $49^{\circ}$ ). Important is the fact that the distribution of the angle on the measured surface changes after the annealing process [16]. Additionally, we present in Fig. 3 the distribution of the molecular form for $\mathrm{CoPc}$ thin layers before and after annealing process.

In our case (300 $\mathrm{nm}$ thick film), dominant form of molecular orientation is $\beta$ orientation. This feature can be explained in terms of different molecule-substrate and molecule-molecule interactions. For various growth processes, the strength of molecule-substrate and molecule-molecule interactions is a crucial parameter which can be changed by different chemical substitutions. The metal-substrate interaction is particularly crucial for the first few layers of the molecules on the metal substrate, whereas for higher coverages the molecule-substrate interaction becomes negligible. Thus, for thicker layers we obtain $\beta$ polymorphic form, where the dominant interaction is moleculemolecule interaction.

In order to compare the results obtained from Raman mapping before and after the annealing process the AFM studies for $\mathrm{CoPc}$ film were performed. AFM images were made for an area of $10 \mu \mathrm{m}^{2} \times 10 \mu \mathrm{m}^{2}$. The analysis of AFM images was carried out using Gwydion 2.33 software. The results achieved for a studied film before and after annealing process are shown in Fig. $4 \mathrm{a}$ and Fig. 4b, respectively. To investigate the structure of the thin layer, couple of parameters evaluated from AFM images were used. Root mean square (RMS)

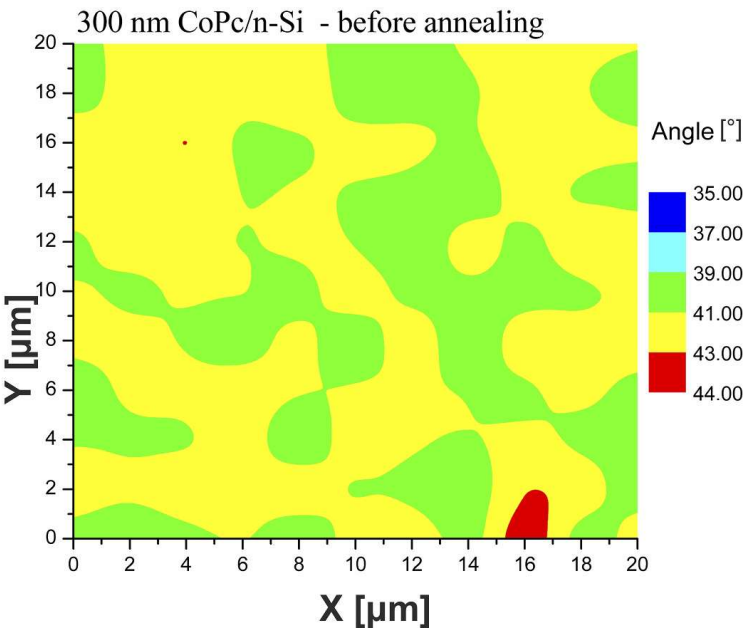

(a)

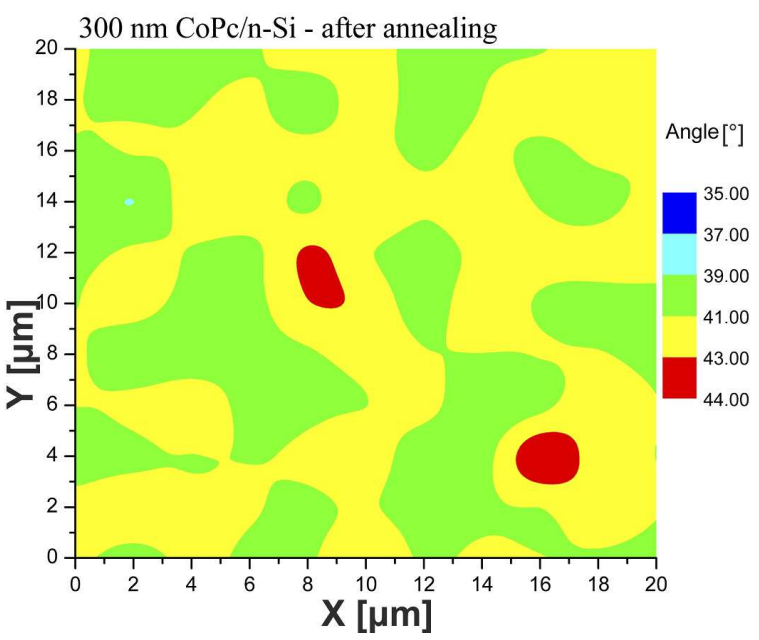

(b)

Fig. 2. Orientation of CoPc molecules (angle between molecule and substrate) obtained from Raman measurements (a) before and (b) after annealing process for $300 \mathrm{~nm}$ thick $\mathrm{CoPc}$ film.

roughness was calculated at 6.37 and $20.8 \mathrm{~nm}$ before and after annealing, respectively. Almost threefold increase of RMS roughness is probably caused by the presence of oxygen during the annealing process $[17,18]$.

The annealing process was carried out in air. Annealing of a film leads to oxidation of cobalt phthalocyanine with adsorbed oxygen. In the annealed films, the rod like crystals deform and become small particles as a result of the oxidation of MPc with adsorbed oxygen. Therefore, 


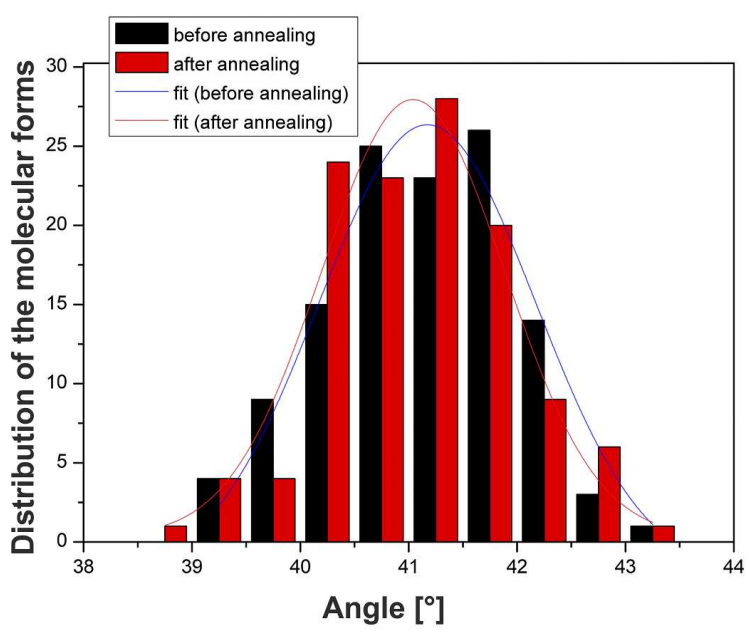

Fig. 3. Distribution of the molecular forms for $\mathrm{CoPc}$ thin layer obtained from Raman measurements before (black) and after (red) annealing.

the formation of the fine particles may proceed as a result of the interaction between the absorbed oxygen and phthalocyanine. This in turn, increases the porosity and, thus, the surface roughness, which is observed by AFM technique. It shows the $\beta$ phase films which consist of larger grain size than before the annealing process.

Despite this fact, the average grain size increased from $71.37 \mathrm{~nm}$ (before annealing) to $83.97 \mathrm{~nm}$ (after annealing) [19]. As can be seen in Fig. 4, very well defined globular structures are observed, which can be connected with similar results from our previous work [20]. After the annealing process, we obtained more homogenous structure, where the grains (with larger diameter) form aggregates and flake-like structure. Prolonged annealing process caused an increase in the value of the parameters characterizing the surface which are collected in Table 1. In this Table we have compiled the most important parameters characterizing surface, obtained from AFM of CoPc images recorded before and after annealing process such as: grain size, average roughness, roughness RMS, average height, maximum height, ratio of average height to maximum height.

Fig. 5 shows the current density-voltage characteristics (J-V) of $\mathrm{Au} / \mathrm{CoPc} / \mathrm{n}-\mathrm{Si} / \mathrm{Al}$ structure under forward and reverse bias, performed under

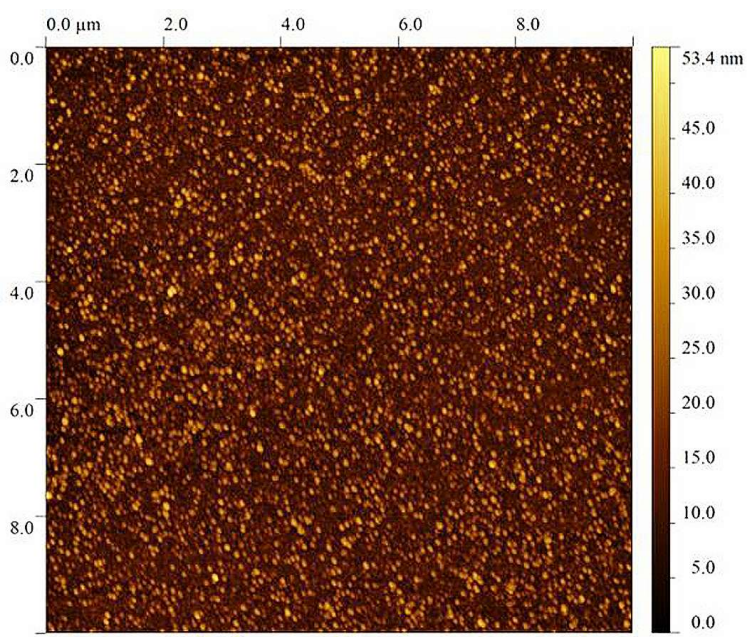

(a)

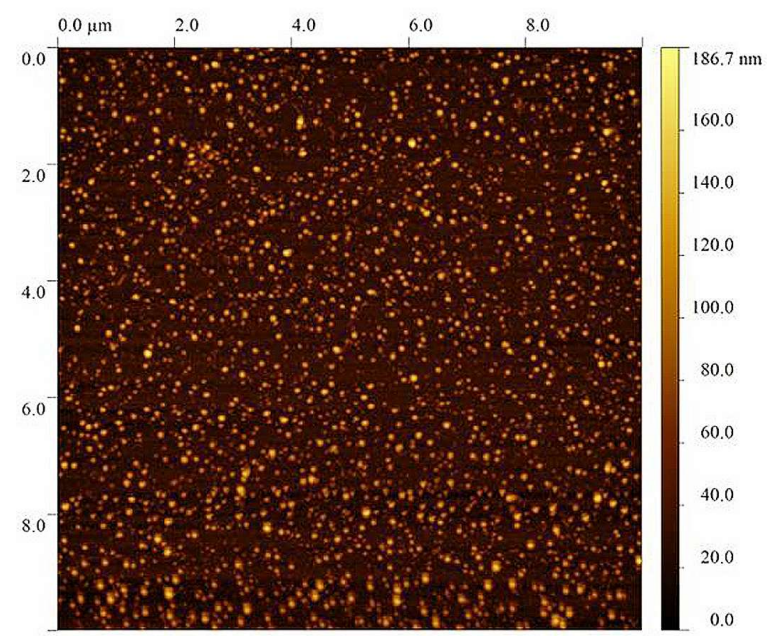

(b)

Fig. 4. AFM images for CoPc thin layer obtained for a) before and $b$ ) after annealing process.

dark current conditions before annealing process in temperature range of $216 \mathrm{~K}$ to $432 \mathrm{~K}$ within voltage range $-5 \mathrm{~V}$ to $+5 \mathrm{~V}$. Fig. 6 shows current-voltage characteristics (I-V) of $\mathrm{Au} / \mathrm{CoPc} / \mathrm{n}$ $\mathrm{Si} / \mathrm{Al}$ before and after annealing process obtained at room temperature [21]. The series resistance $\left(R_{s}\right)$ and the shunt resistance $\left(\mathrm{R}_{\mathrm{sh}}\right)$ are directly related to the device performance, determined from the plot of the junction resistance $\left(\mathrm{R}_{\mathrm{j}}\right)$ against voltage where:

$$
R_{j}=\partial V / \partial I
$$


Table 1. The surface parameters obtained from AFM images for CoPc thin film before and after annealing process.

\begin{tabular}{ccccccc}
\hline$[\mathrm{nm}]$ & Grain size & Ra & RMS & Avg. height & Max. height & Avg./Max. \\
\hline \hline before annealing & $71.37 \pm 14.65$ & 4.99 & 6.37 & 13.01 & 53.40 & 0.244 \\
after annealing & $83.97 \pm 7.97$ & 14.50 & 20.80 & 32.40 & 186.70 & 0.174 \\
\hline
\end{tabular}

which was determined from the current-voltage characteristics and $R_{j}$ plotted versus voltage. The series and shunt resistances $\left(\mathrm{R}_{\mathrm{s}}\right.$ and $\left.\mathrm{R}_{\mathrm{sh}}\right)$, which are important factors in improving cell performance and design, obtained at room temperature by the method presented in the literature [22], using the relation between junction resistance, $R_{j}$, and the applied voltage, V, are shown in Fig. 7.

As observed in Fig. 7, at sufficiently high forward bias, the junction resistance approaches a constant value characterizing the series resistance $\mathrm{R}_{\mathrm{s}} \approx 5.2 \mathrm{k} \Omega$. In addition, in Fig. 7, the junction resistance shows almost constant value at sufficiently high reverse bias, which equals the diode shunt resistance $\mathrm{R}_{\mathrm{sh}} \approx 5.69 \mathrm{M} \Omega$.

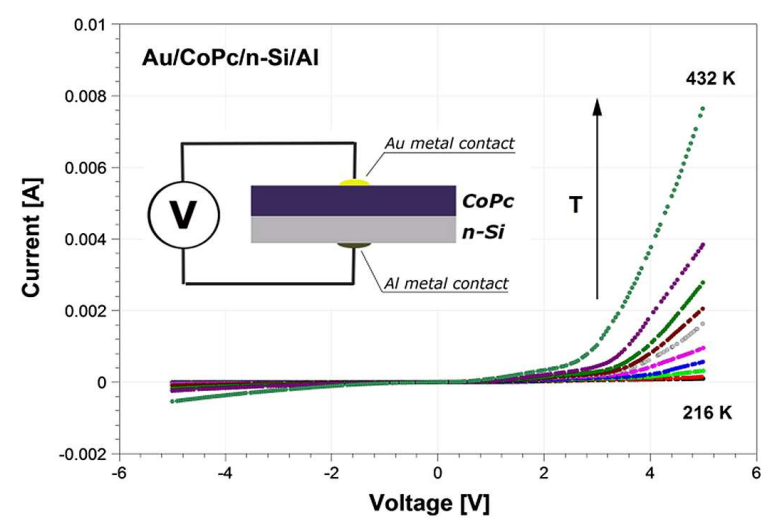

Fig. 5. Current-voltage characteristics (I-V) for CoPc thin layers before annealing; inset in Fig. 5 shows the electrical diagram.

Thus, we can observe changes in current characteristics due to the annealing process of thin film structure. This is probably due to the reorientation of the molecules and alignment of the molecular structure of the organic thin film during long time annealing process. It is known from our previous work that prolonged annealing process causes changes in the polymorphic forms of MPc thin layer structure $[14,15]$.

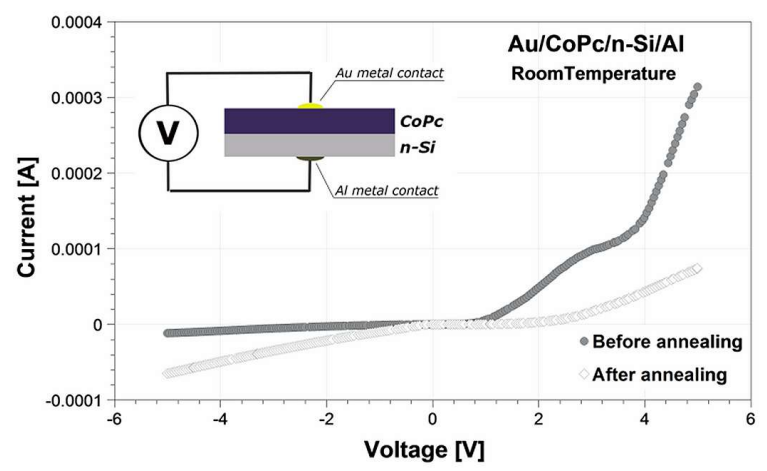

Fig. 6. Current-voltage characteristics (I-V) for CoPc thin layers before and after annealing process at the temperature of $473 \mathrm{~K}$ for 6 hours in air; the inset in Fig. 6 shows the electrical diagram.

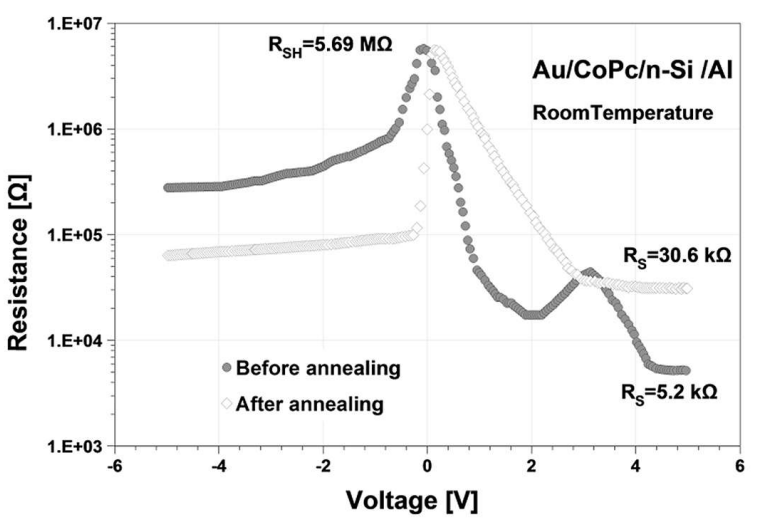

Fig. 7. Plots of junction resistance, $R_{j}$, against applied voltage at room temperature for $\mathrm{Au} / \mathrm{CoPc} / \mathrm{n}$ $\mathrm{Si} / \mathrm{Al}$ structure annealed at temperature of $473 \mathrm{~K}$ for 6 hours in air.

Using Cheung's functions and thermionic emission theory [23, 24] describing the currentvoltage characteristics and the resulting electrical parameters for semiconductors, we calculated electrical parameters of the $\mathrm{Au} / \mathrm{CoPc} / \mathrm{n}-\mathrm{Si} / \mathrm{Al}$ structure such as series resistance and potential barrier for $\mathrm{Au} / \mathrm{CoPc} / \mathrm{n}-\mathrm{Si} / \mathrm{Al}$ structure before and after annealing process. These parameters are shown in Table 2. 
The electrical conductivity of phthalocyanines is very sensitive to the presence of adsorbed oxygen in the organic thin films. The annealing process in air causes adsorption of oxygen on the surface of the thin film. Adsorbed oxygen causes creation of the depletion region and next oxygen diffuses into the crystalline organic film. This, in turn, increases surface roughness. This fact can be ascribed to the increase in the conductivity of CoPc layer induced by oxygen adsorption, which causes a change of the capacity of the thin layer. The model proposed here assumes that oxygen molecules diffuse into the $\mathrm{CoPc}$ film and act as electron acceptor sites. In this case, we can observe drastic changes in capacitance-voltage curves and change in electrical properties of the thin layers [25]. Thus, electrical conductivity and surface topography is closely linked to the amount of oxygen adsorbed in the thin film.

Table 2. Electrical parameters obtained from I-V characteristic for $\mathrm{CoPc}$ structure, measured at room temperature in dark.

\begin{tabular}{ccc}
\hline $\mathrm{Au} / \mathrm{CoPc} / \mathrm{n}-\mathrm{Si} / \mathrm{Al}$ & $\begin{array}{c}\text { Series resistance } \\
\text { of the structure }[\Omega]\end{array}$ & $\begin{array}{c}\text { Potential } \\
\text { barrier }[\mathrm{eV}]\end{array}$ \\
\hline \hline $\begin{array}{c}\text { before annealing } \\
\text { after annealing }\end{array}$ & 5200 & 0.79 \\
\hline
\end{tabular}

The dark current-voltage measurements suggest that the forward current transport in these heterojunctions involves thermionic emission of electrons from $\mathrm{n}-\mathrm{Si}$ over the $\mathrm{CoPc} / \mathrm{Si}$ barrier at low forward bias. Moreover, a space-charge-limited transport across the CoPc layer dominates due to the exponential distribution of traps above the valence band in the band gap of the CoPc layer at high forward bias.

\section{Conclusions}

In this paper we performed the orientation study of $\mathrm{CoPc}$ thin layers deposited on silicon substrate using micro-Raman scattering spectroscopy, AFM measurement and I-V characteristics. It has been shown, that the Raman scattering method, atomic force microscopy (AFM) and current-voltage (I-V) characteristics are very useful to investigate molecular orientation and electrical parameters of molecules deposited on a solid substrate as a potential molecular devices. We have studied how the annealing process affects the optical and electrical characteristics of organic thin film structures. The obtained results showed that the annealing process of the thin layer causes molecular ordering and thus improves the electrical properties of organic thin film. The knowledge of derived parameters is important for modification of the technological process for obtaining thin film structures. From obtained results it can be shown that annealing process improves the structural and electrical properties of the structures. The extracted diode parameters are consistent with those obtained from the techniques of Cheung for organic thin films deposited on Si substrate. It was revealed that annealing process affects the structural organization and homogeneity of organic thin layers. A better description of electrical parameters connected with the orientation and polymorphic form of organic thin layers requires further studies which will be carried out in future.

\section{Acknowledgements}

The Project was supported through the European Union - European Social Fund and Human Capital - National Cohesion Strategy (RS) and in part by the Research Project of Poznan University of Technology 06/65/DSPB/0516.

\section{References}

[1] Simon J., Andre J.J., Molecular Semiconductors, Springer Verlag, New York, 1985.

[2] HANACK M., Turk. J. Chem., 22 (1998), 13.

[3] Kerp H.R., FaAssen van E.E., Chem. Phys. Lett., 332 (2000), 5.

[4] CARPi F., Rossi De D., Opt. Laser Technol., 38 (2006), 292.

[5] Kobayashi N., Curr. Opin. Solid St. M., 4 (1999), 345.

[6] Granito C., Goldenberg L.M., Bryce M.R., Monkman A.P., Troisi L., Pasimeni L., Petty M., Langmuir, 12 (1996), 472.

[7] Hanack M., LANG M., Adv. Mater, 6 (1994), 819.

[8] BrożeK-PŁuska B., Jarota A., Kurczewski K., ABRAMCZYK H., J. Mol. Struct., 924 - 926 (2009) 338.

[9] Kolesov B.A., Basova T.V., Thin Solid Films, 304 (1997), 166.

[10] Zawadzka A., PŁóciennik P., Strzelecki J., Pranaitis M., Dabos-Seignon S., Sahraoui B., Thin Solid Films, 545 (2013), 429.

[11] Zawadzka A., PŁóciennik P., Strzelecki J., KoRCAla A., Arof A.K., Sahraoui B., Dyes Pigments, 101 (2014), 212. 
[12] Tackley D.R., Dent G., Smith W.E., Phys. Chem. Chem. Phys., 3 (2001), 1419.

[13] Szybowicz M., Runka T., Drozdowski M., BAŁA W., WojdyŁa M., Grodzicki A., PiszczeK P., BratKowski A., J. Mol. Struct., 830 (2007), 14.

[14] Szybowicz M., Runka T., Drozdowski M., BAŁA W., GRODZICKI A., PISZCZEK P., BratKowski A., J. Mol. Struct., 704 (2004), 107.

[15] Szybowicz M., BaŁa W., FabisiaK K., PAPROCKI K., DROZDOWSKI M., Cryst. Res. Technol., 45 (2010), 1265.

[16] Shinub S.I., Gould R.D., Phys. Status Solidi A, 139 (1993), 129.

[17] Dijken VAn J.G., Brett M.J., Molecules, 17 (2012), 10119.

[18] Shinub S.I., Gould R.D., Gravano S., Physica B, 222 (1996), 136.
[19] Hussein M.T., Nasir E.M., Kasim T., Senaed F.A., Int. J. Cur. Eng. T., 4 (2014), 3263.

[20] Szybowicz M., Makowiecki J., J. Mater. Sci., 47 (2012), 1522.

[21] Soliman H.S., El-Barry A.M.A., Khosifan N.M., El Nahass M.M., Eur. Phys. J-Appl. Phys., 37 (2007), 1.

[22] Darwish S., El-Zawawi I.K., Riad A.S., Thin Solid Films, 485 (2005), 182.

[23] Cheung S.K., Cheung N.W., Appl. Phys. Lett., 49 (1986), 85

[24] Wahab F., Sayyad M.H., Khan D.N., TAhir M., AzIz F., Shahid M., Munawar, M.A., Chaudry J.A., Mat. Sci. Semicon. Proc., 26 (2014), 101.

[25] Buchholtz J.C., Appl. Surf. Sci., 1 (1978), 547.

Received 2016-02-22 Accepted 2016-07-09 